\title{
Case Report \\ Hypertrophic Osteodystrophy in Two Red Wolf (Canis rufus) Pups
}

\author{
Jenessa L. Gjeltema, ${ }^{1,2,3}$ Robert A. MacLean, ${ }^{3,4}$ Eli B. Cohen, ${ }^{1}$ and Ryan S. De Voe ${ }^{3,5}$ \\ ${ }^{1}$ Departments of Clinical and Molecular Biomedical Sciences, College of Veterinary Medicine, North Carolina State University, \\ 1060 William Moore Drive, Raleigh, NC 27607, USA \\ ${ }^{2}$ North Carolina Zoo, Asheboro, NC 27205, USA \\ ${ }^{3}$ Environmental Medicine Consortium, College of Veterinary Medicine, North Carolina State University, Raleigh, NC 27207, USA \\ ${ }^{4}$ Audubon Nature Institute, 6500 Magazine Street, New Orleans, LA 70118, USA \\ ${ }^{5}$ Disney's Animal Kingdom, Orlando, FL 32830, USA
}

Correspondence should be addressed to Jenessa L. Gjeltema; jenessa_gjeltema@ncsu.edu

Received 27 February 2015; Accepted 28 April 2015

Academic Editor: Sheila C. Rahal

Copyright ( 2015 Jenessa L. Gjeltema et al. This is an open access article distributed under the Creative Commons Attribution License, which permits unrestricted use, distribution, and reproduction in any medium, provided the original work is properly cited.

A 6-month-old red wolf (Canis rufus) pup presented for evaluation of progressive thoracic and pelvic limb lameness, joint swelling, and decreased body condition. Radiographic evaluation revealed medullary sclerosis centered at the metaphyses of multiple long bones, well-defined irregular periosteal proliferation, and ill-defined lucent zones paralleling the physes, consistent with hypertrophic osteodystrophy (HOD). Biopsies of affected bone revealed medullary fibrosis and new bone formation. The pup improved following treatment with nonsteroidal anti-inflammatories, opioids, and supportive care over the course of 4 weeks. Metaphyseal periosteal bone proliferation persisted until the animal was humanely euthanized several years later for poor quality of life associated with bilateral cranial cruciate ligament rupture. A second red wolf pup of 4.5 months of age presented for evaluation of lethargy, kyphotic posture, and swollen carpal and tarsal joints. Radiographs revealed bilateral medullary sclerosis and smooth periosteal reaction affecting multiple long bones, suggestive of HOD. Further diagnostics were not pursued in this case to confirm the diagnosis, and the clinical signs persisted for 4 weeks. In light of these two case reports, HOD should be recognized as a developmental orthopedic disease in growing red wolves.

\section{Introduction}

Hypertrophic osteodystrophy (HOD), also referred to as metaphyseal osteopathy, is a developmental disease affecting the metaphyses of bones in young growing animals. Altered vascularity, necrosis, suppurative inflammation, and modeling of bone at the affected metaphyses have been described $[1,2]$. Affected animals may exhibit signs of discomfort, lameness, and general malaise related to the condition. Nutritional, infectious, vaccine-associated, and congenital causes have been implicated in the development of this disease; however, the exact pathogenesis remains unknown [3-9]. The disease has been well documented in domestic canines (Canis lupus familiaris), and there have also been reports of the disease in domestic cats (Felis domesticus) and Iberian lynx (Lynx pardinus) [10-12].
The International Union for the Conservation of Nature (IUCN) currently lists the red wolf as critically endangered [13]. While once considered extinct in the wild in 1980, reintroduction programs have since established a small population in the Southeastern United States of America. This report describes the presentation, diagnosis, and management of HOD in one red wolf (Canis rufus) pup and the presentation and management of suspected HOD in a second pup.

\section{Case 1}

A captive bred male red wolf pup of 6 months of age presented for evaluation of progressive lameness of the thoracic and pelvic limbs, joint swelling, and decreased body condition over the course of nine days. The animal was housed with six other conspecifics including four littermates in a natural 


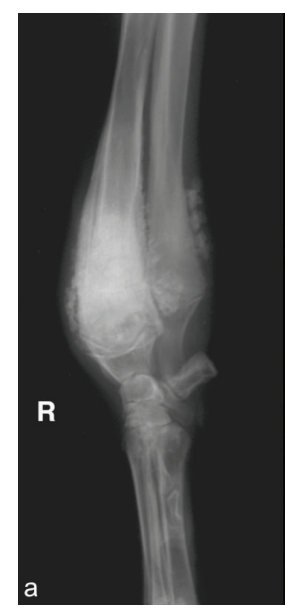

(a)

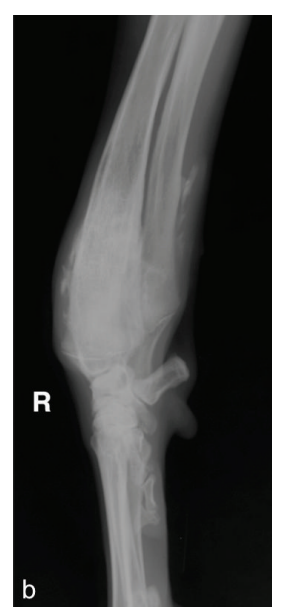

(b)

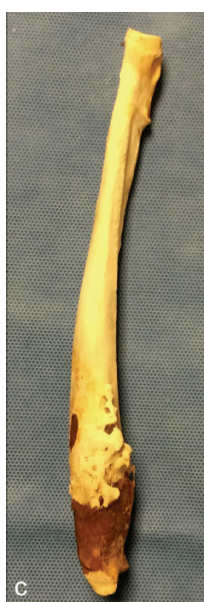

(c)

FIGURE 1: Radiographs and gross photograph obtained of the right distal limb from the red wolf pup of case 1 depicting characteristic bone lesions consistent with hypertrophic osteodystrophy. (a) Lateral radiograph day 9 . The distal radial and ulnar metaphyses are distally flared with severe, well-defined, and irregular to palisading periosteal bone formation and medullary sclerosis. An irregular zone of lucency is present proximal to the distal radial physis. (b) Lateral radiograph day 62. There is reduction of the prior distal radial and ulnar periosteal bone formation and medullary sclerosis. The distal radial subphyseal linear lucency is no longer present. (c) Postmortem gross image of the right radius demonstrating well-defined, irregular periosteal bone formation at the metaphysis.

substrate outdoor enclosure with access to several den boxes. All wolves were fed Mazuri ${ }^{\circledR}$ exotic canine formulated diet \#5MN2 (Mazuri ${ }^{\circledR}$, PMI $^{\circledR}$ Nutrition International, Inc., Brentwood, MO 63144, USA) ad libitum and were supplemented with a moist canned food temporarily at the time of weaning. There were no reported complications during parturition. The pup was treated for ascariasis but had been normal with an unremarkable medical history up to the time of presentation. It had received preventive care including periodic deworming and routine vaccinations, and none of its littermates demonstrated clinical signs.

The animal was immobilized to perform a full physical examination and diagnostics using midazolam (Akorn Inc., Lake Forest, IL 60045, USA; $0.17 \mathrm{mg} / \mathrm{kg}$ ) intramuscularly followed by mask-induction with isoflurane gas. On physical examination, the pup had a thin body condition at a weight of $14 \mathrm{~kg}$ and was noticeably smaller than its littermates. Swelling with associated heat was present bilaterally at the carpi, tarsi, and stifle joints. All joints had normal range of motion with no crepitus palpated during flexion or extension.

A complete blood count and serum biochemistry were performed and compared to values listed for red wolves in the International Species Information System (ISIS) database [14]. There was marked eosinophilia $\left(4,765 \times 10^{6}\right.$ cells/L; ISIS values 144-2,393) consistent with underlying endoparasitism, a decreased blood urea nitrogen $(3.2 \mathrm{mmol} / \mathrm{L}$; ISIS values 3.8-14.4) possibly related to the animal's young age or a decreased intake of dietary protein, and hyperphosphatemia $(2.45 \mathrm{mmol} / \mathrm{L}$; ISIS values $0.62-2.28)$ with elevated alkaline phosphatase (319 U/L; ISIS values 7-73) consistent with growth in a young animal. Fecal floatation analysis was performed with sodium nitrate solution, which revealed occasional ova consistent with Toxocara and Ancylostoma sp.
Differential diagnoses included hypertrophic osteodystrophy, septic arthritis, and osteomyelitis.

Orthogonal screen-film radiographs were obtained (Figure 1(a)). Centered at the metaphyses and extending into the diaphyses of multiple long bones bilaterally (proximal humeri, proximal and distal radii, distal ulnae, distal femur, and proximal and distal tibiae and fibulae) increased medullary opacity was present resulting in loss of visualization of trabeculation, consistent with sclerosis. At these sites, there was also varying degrees of well-defined, irregularly marginated periosteal bone formation resulting in flaring of the metaphyses, which was palisading in some regions. The periosteal change was most severe at the distal antebrachia as well as the proximal and distal aspects of the crura. Additionally, within the proximal humeri, distal radii and ulnae, and proximal and distal tibiae, ill-defined irregularly linear zones of lucency were present within the metaphyseal bone, which paralleled the physes. The adjacent physes were well defined. Within the proximal diaphysis of the left ulna, faint, ill-defined, and increased medullary opacity was present. Complete evaluation of soft tissues at all sites was hindered due to technique, but concurrent soft-tissue swelling at these sites was suspected. The primary differential for the polyostotic change centered at the metaphyses of multiple long bones was HOD. Concurrent osteomyelitis was also considered. The diaphyseal sclerosis within the left proximal ulna was most consistent with panosteitis or other causes of bone infarction.

The pup was treated supportively with buprenorphine (American Regent, Inc., Shirley, NY 11967, USA; 0.008 mg/kg) subcutaneously, meloxicam (Metacam ${ }^{\circledR}$, Boehringer Ingelheim Vetmedica, Inc., St. Joseph, Mo 64506, USA; 0.2 mg/kg) 
intramuscularly, and a fentanyl transdermal patch (Duragesic $^{\circledR}$, ALZA corp., Vacaville, CA 95688, USA; 50 mcg/hr). Meloxicam was also prescribed $(0.1 \mathrm{mg} / \mathrm{kg})$ orally once daily for 10 days and a single dose of pyrantel pamoate (Strongid $\mathrm{T}^{\circledR}$, Pfizer Inc., New York, NY 10017, USA; $32 \mathrm{mg} / \mathrm{kg}$ ) orally. Over the next week, the pup maintained an appetite; however, its lameness and joint swelling persisted.

A follow-up procedure was performed on day 18 after the onset of clinical signs. The animal was anesthetized using midazolam $(0.2 \mathrm{mg} / \mathrm{kg})$ and buprenorphine $(0.01 \mathrm{mg} / \mathrm{kg})$ intramuscularly followed by mask induction with isoflurane gas. On physical examination there was progressive swelling of the previously affected joints. These joints were subjectively less warm to the touch than at the original evaluation of the animal. A complete blood count showed resolution of the previous eosinophilia $\left(1,420 \times 10^{6}\right.$ cells/L; ISIS values $144-$ 2,393). A serum biochemistry revealed a decreased blood urea nitrogen $(1.79 \mathrm{mmol} / \mathrm{L}$; ISIS values 3.8-14.4), hyperphosphatemia $(2.49 \mathrm{mmol} / \mathrm{L}$; ISIS values $0.62-2.28)$, and elevated alkaline phosphatase activity (103 U/L; ISIS values 7-73). Orthogonal radiographs of the thorax, abdomen, and shoulders were performed, which showed a static appearance of the proximal humeral metaphyseal sclerosis and subphyseal lucency. No thoracic or abdominal abnormalities were identified radiographically.

Bone biopsies were performed at the metaphyses of the distal left radius and the right proximal tibia using aseptic technique. Two 15 ga core biopsies were obtained from each site and placed into $10 \%$ buffered formalin for histopathologic evaluation. Following the procedure, meloxicam $(0.15 \mathrm{mg} / \mathrm{kg})$ was administered intramuscularly. Recovery from anesthesia was uneventful. Three additional blood samples were collected under manual restraint for aerobic and anaerobic bacterial culture on days 20,23 , and 25 using aseptic technique.

Histopathology of the bone biopsies indicated diffuse moderate medullary fibrosis with new bone formation. No signs of active inflammation were present in the submitted samples. No bacterial growth was present after 14 days from the first two blood cultures; however, Clostridium bifermentans was cultured from the final sample. Due to this positive blood culture result, a complete blood count, serum biochemistry, and an additional follow-up blood culture sample were obtained on day 35 . The complete blood count revealed a mild monocytosis $\left(1,803 \times 10^{6}\right.$ cells/L; ISIS values $\left.87-1,214\right)$ indicative of chronic inflammation. A serum biochemistry revealed a decreased blood urea nitrogen $(3.57 \mathrm{mmol} / \mathrm{L}$; ISIS values 3.8-14.4), hyperphosphatemia $(2.65 \mathrm{mmol} / \mathrm{L}$; ISIS values $0.62-2.28$ ), and elevated alkaline phosphatase activity (103 U/L; ISIS values 7-73) similar to the animal's previous results. Because Clostridium bifermentans is only rarely reported to cause osteomyelitis in children and elderly humans, it was presumed to be an environmental contaminant [15]. A follow-up fecal analysis revealed no evidence of parasites.

Caretakers reported marked improvement of the animal's gait and ability to move by day 28 , with no additional episodes of lameness through day 60. A physical examination was performed on day 62 in preparation for transfer of the animal to another facility. Radiographs were repeated (Figure 1(b)). Metaphyseal and diaphyseal medullary scleroses as well as periosteal bone formation remained at the prior sites but were markedly reduced. An additional similar lesion to the other long bones was present within the second metatarsal bilaterally. This region was not imaged on prior dates and this may have been present previously as opposed to being a novel lesion. The prior palisading periosteal bone production and subphyseal lucent zones were no longer present. The prior left ulnar proximal diaphyseal sclerosis was also no longer present. The imaged physes remained open.

For the following two years, periodic physical examinations were performed, which demonstrated persistent bilateral carpal and stifle swelling. At 30 months of age, the animal developed severe right pelvic limb lameness. Based on physical examination and stifle radiography, cranial cruciate ligament rupture with associated stifle osteoarthrosis was diagnosed. The animal was treated with restricted activity, nonsteroidal anti-inflammatory medications, and supplementation with glucosamine and chondroitin. The lameness improved but did not completely resolve. At 34 months of age, the animal developed severe bilateral pelvic limb lameness. Physical examination and radiographic findings were suggestive of bilateral cranial cruciate ligament rupture and associated osteoarthrosis. These radiographs were not available for review by the authors of this case report. The animal was not responsive to medical management with restricted activity and administration of nonsteroidal anti-inflammatory medications. Due to a poor overall quality of life related to the lameness, humane euthanasia was elected.

Gross necropsy confirmed the diagnosis of bilateral cranial cruciate ligament rupture. There were defects of the articular cartilage on the lateral femoral condyle and osteophytosis of both stifles. Thickening of the lateral collateral ligaments and joint capsules of both stifles was also noted. Moderate medial and lateral flaring of the metaphyses with periosteal proliferation of bone was noted at the distal radii (Figure 1(c)). No other musculoskeletal abnormalities were found, and other than several missing teeth, the remainder of the necropsy findings were normal.

\section{Case 2}

A captive-bred male red wolf pup of 4.5 month of age presented as an emergency for a rectal prolapse that failed to resolve with medical management. The wolf was housed with 5 siblings, and their diet consisted of Science Diet Canine Adult Active formula (Hills Pet Nutrition Inc., Topeka, KS 66603, USA), carnivore diet (Bravo Packing, Inc., Penns Grove, NJ 08069, USA), and occasional rats or mice. It had received preventive care including periodic deworming and routine vaccinations, and none of its littermates demonstrated clinical signs. A cecal inversion was diagnosed by ultrasound, and an exploratory laparotomy was subsequently performed. An ileocolic intussusception with cecal inversion was discovered. A subtotal colectomy and distal ileectomy with ileocolic anastomosis was performed, and the animal recovered uneventfully. No other abnormalities were noted at this time. 
Approximately 2 weeks later, the wolf presented for lethargy and a kyphotic posture, presumed to be related to abdominal pain. The animal was examined under sedation with medetomidine (Domitor ${ }^{\circledR}$, Pfizer Inc.; $0.05 \mathrm{mg} / \mathrm{kg}$ ) intramuscularly and was administered intravenous crystalloid fluids, ticarcillin disodium, and clavulanate potassium (Timentin $^{\circledR}$, GlaxoSmithKline, Inc., Philadelphia, PA 19112, USA; $30 \mathrm{mg} / \mathrm{kg}$ IV $\mathrm{q} 6 \mathrm{~h}$ for $2 \mathrm{~d}$ ) and meloxicam $(0.1 \mathrm{mg} / \mathrm{kg}$ IM, $\mathrm{q} 24 \mathrm{~h}$ for $2 \mathrm{~d})$. His temperature was elevated $\left(41.1^{\circ} \mathrm{C}\right)$ after manual restraint and sedation. Abdominal and thoracic radiographs as well as a complete blood count were considered unremarkable. The animal was discharged on oral antibiotics (amoxicillin clavulanate, Clavamox ${ }^{\circledR}$, Pfizer Inc.; $250 \mathrm{mg}$ PO q12 h for $14 \mathrm{~d}$ ) and oral meloxicam daily, as needed.

The animal's kyphotic posture worsened 2 weeks after surgery and another examination under anesthesia was conducted at four weeks after surgery. All complete blood count and serum biochemistry results were within species values reported by ISIS, other than a mild monocytosis $(1577 \times$ $10^{6}$ cells/L; species values 87-1,214). An abdominal ultrasound revealed a suspected gastrointestinal foreign body, and an exploratory laparotomy was performed, which was considered to be within normal limits. Carpal and tarsal joints were noted to be swollen bilaterally during the exam. Orthogonal view radiographs of the thorax, abdomen, hips, and distal limbs obtained during the procedure were submitted to a consulting radiology service (Insight Radiology, San Diego, CA 92111, USA). Centered at the diaphysis and metaphyses of the distal femurs, radii, and ulnae bilaterally, smooth, welldefined periosteal bone formation was present. Medullary sclerosis was also identified. Similar lesions were noted in the proximal humerus, bilaterally. The distal aspects of the left 8th and 10th ribs were flared and were more lucent than adjacent ribs. The radiographic appearance was suggestive, but not specific for a systemic disorder of cartilage formation or cartilage inflammation characteristic of HOD. These images were not available for further review by the authors of this case report.

The animal was managed successfully with oral meloxicam for the next 3 months. During this time, whenever antiinflammatory therapy was discontinued, the animal would become anorexic. After 3 months, his treatment was then tapered and discontinued successfully. The animal's adult weight was $32.5 \mathrm{~kg}$. He died acutely of unrelated causes at 20 months of age. Histopathology of the bones was not pursued at the time of necropsy.

\section{Discussion}

Hypertrophic osteodystrophy is an important developmental disease that affects the bones of growing animals. This report describes one confirmed and one suspected case of hypertrophic osteodystrophy in two red wolf pups, which to the author's knowledge has not been previously reported in this species. In the first case, a diagnosis of HOD in multiple long bones with concurrent panosteitis at the left ulna was made based on clinical history, radiographic findings, bone biopsy, and negative blood cultures. Hypertrophic osteodystrophy was also suspected in the second wolf pup based on radiographic findings, although additional diagnostics were not performed to confirm the diagnosis. The onset of disease for the red wolf pups of this report occurred between 5 and 6 months of age. This is consistent with what is frequently seen in domestic dogs (Canis familiaris), with pups under 6 months of age considered to be at the highest risk of developing the disease [16]. Common clinical signs in domestic dogs include pyrexia, lethargy, anorexia, lameness, soft-tissue swelling at affected bones, and ostealgia [8]. The severity of disease varies, and some cases have episodic recurrence of clinical signs. The presentation of both red wolf pups was similar to that seen in domestic canines and included lameness and soft-tissue swelling. Although anorexia was not documented in the first case due to the presence of several other conspecifics within its shared enclosure, a degree of anorexia or decreased appetite was likely in considering the animal's thin body condition at presentation.

Long bones including the radius, ulna, and tibia are most commonly affected in dogs, and the disease is usually bilateral. Radiographs of the red wolves described in this report revealed involvement of many of the long bones, with the radii, ulnae, and tibias most severely affected. Early radiographic abnormalities of affected bones may include radiolucent zones within the metaphyses parallel to the physis as well as soft-tissue swelling. As the disease progresses, there may be increased metaphyseal medullary opacity along with periosteal or extraperiosteal new bone formation $[1,4]$. In some cases, development of clinical signs may precede radiographic abnormalities. Increased metaphyseal medullary opacity with associated periosteal new bone formation was seen in both red wolf pups. These radiographic findings indicated chronicity, seen in later stages of HOD. The subphyseal lysis that was concurrently seen in the first case may represent incomplete resolution of the initial stages of disease or an acute on chronic occurrence. The earliest stages of the disease in the red wolf pups were not captured radiographically. This is likely due to delay between initial onset of clinical signs and acquisition of radiographs.

Treatment of this disease is symptomatic and supportive with specific therapies directed at alleviation of pain, inflammation, and pyrexia associated with the condition. Nonsteroidal anti-inflammatory medications or steroids are appropriate and effective for treatment of HOD [8], and crystalline fluid therapy, nutritional support, opioid pain medications, and other supportive care measures may also be clinically indicated in some cases. The wolf of the first case report responded favorably to treatment with nonsteroidal antiinflammatory medications, opioids, and intermittent crystalline fluid therapy. The wolf in the second case responded well to nonsteroidal anti-inflammatory therapy alone.

Clinical signs can persist for days to weeks in domestic canines with relapses occurring in some individuals. In the first case, clinical improvement occurred 4 weeks following initial presentation, and no relapses occurred following recovery. Despite clinical improvement, the animal of the first case report had residual skeletal abnormalities at affected bones that persisted throughout its life. These abnormalities 
were evident on the preserved skeletal remains from this animal. Reports in the literature indicate that some animals with developmental orthopedic disease may also experience subsequent cranial cruciate ligament rupture [17]. While plausible, it is not known if the residual skeletal abnormalities seen in the first case contributed to the development of bilateral cranial cruciate ligament rupture several years later. This case was considered severe due to the extent of the lesions, number of affected bones, the lengthy clinical course, and the persistent proliferative bone lesions that did not resolve despite clinical improvement. Although these factors are suggestive of a severe case, clinical signs indicating pain were subtle and less apparent than expected. This may be due to behavioral differences between domestic dogs and red wolves. Clinical signs for the wolf in the second case appeared to resolve after 3 months of therapy and residual lesions were not clinically apparent; however, no histologic evidence was obtained postmortem.

Excessive dietary calcium, phosphorous, protein, and energy have been identified as factors leading to developmental orthopedic diseases in dogs and could play a role in the development of $\operatorname{HOD}[18,19]$. The specific nutritional requirements for red wolves are unknown and the current husbandry guidelines are based on recommendations for domestic dogs, including feeding a high quality commercial dry dog food with additional supplementation provided at and following whelping [20]. Use of a commercial growth formulation for red wolf pups is not currently recommended due to the feeding challenges related to managing red wolves in family groups that minimize habituation to humans. The average weight of adult male red wolves is $27.6 \mathrm{~kg}$ [21], which is comparable in size to many large breed domestic dogs that are prone to rapid growth and developmental bone disease [6]. The adult weights for the wolves of this case series were $25 \mathrm{~kg}$ for the first case and the $33 \mathrm{~kg}$ for the second case. The wolf of the first case was maintained on a diet consisting predominantly of Mazuri ${ }^{\circledR}$ exotic canine formula provided ad libitum. Ad libitum feeding of large breed dogs has been associated with an increased occurrence of skeletal abnormalities and developmental bone diseases. The diet fed to the wolf of the first case also contains $1.7 \%$ calcium and $0.96 \%$ phosphorous on a dry matter basis and exceeds the dietary calcium recommendations established by the National Research Council for large breed puppies at risk for developmental orthopedic disease [22]. The wolf of the second case was fed Science Diet Adult Active formula, which has 1.04\% calcium and $0.8 \%$ phosphorous on a dry matter basis. This diet is within the recommended range, and the extent to which nutritional factors contributed to the development of hypertrophic osteodystrophy in either case is unknown.

Infection with Canine Distemper Virus (CDV) and vaccination have also been implicated as other potential etiologies for HOD. Viral RNA has been found in the metaphyses of CDV-infected dogs, and a strong correlation has been established between CDV infection and metaphyseal bone lesions from young dogs [7, 23, 24]. Additionally, several reports have described systemic clinical signs similar to those associated with $\mathrm{CDV}$ in cases of $\operatorname{HOD}[2,3,5]$. Although these findings are suggestive of a link between CDV and HOD, clear evidence of a relationship between the two diseases has not been established [16]. Reports have also described its clinical onset occurring several weeks following multiple doses of polyvalent vaccines including modified live CDV, canine adenovirus type 2, and Leptospira bacterin [3,5]. Both pups in this report did receive polyvalent vaccines at routine 2 -3-week intervals from 2 to 4 months of age, but neither pup nor any littermates exhibited gastrointestinal signs, respiratory signs, hyperkeratotic footpads, or neurologic deficits consistent with what is seen with CDV. PCR analysis of bone biopsies for CDV RNA was not performed for either red wolf pup and viral or vaccination-related causes for the development of HOD in these cases cannot be completely ruled out.

It is believed that genetics may be a contributing factor in the development of HOD in some cases. Breed predispositions have been identified in domestic canines $[6,16]$, and familial trends also occur in breeds, such as the Weimaraner $[2,3,5]$. This indicates that heredity may play a large role in the disease for some cases. None of the littermates of the two pups described in this case report exhibited clinical signs of HOD, and familial trends were not identified in these cases. However, an underlying genetic cause of this disease in red wolves remains a possibility and may be of concern for future red wolf breeding recommendations.

Captive breeding programs continue to play a large role in conservation and management efforts for the red wolf. The captive red wolf population consists of about 180 wolves, which was founded from 12 individuals [17]. Understanding disease susceptibility and etiology in red wolves is important for making appropriate and well-informed management decisions that balance the goals of maintaining genetic diversity with the risk of perpetuating potentially genetic diseases within the population. Although the underlying pathogenesis of HOD remains poorly understood, the potential for genetic, nutritional, infectious, and vaccine-associated causes makes it a disease of particular concern and interest for the future management of this species.

\section{Conflict of Interests}

The authors declare that there is no conflict of interests regarding the publication of this paper.

\section{Acknowledgments}

The authors would like to sincerely thank Chris Lasher, Katy Harringer, Sherry Samuels, Kathy Long, the Virginia Living Museum, and the Museum of Life and Science for their cooperation and assistance with this case report.

\section{References}

[1] H. Meier, S. T. Clark, G. B. Schnelle, and D. H. Will, "Hypertrophic osteodystrophy associated with disturbance of vitamin C synthesis in dogs," Journal of the American Veterinary Medical Association, vol. 130, no. 11, pp. 483-491, 1957.

[2] J. C. Woodard, "Canine hypertrophic osteodystrophy, a study of the spontaneous disease in littermates," Veterinary Pathology, vol. 19, no. 4, pp. 337-354, 1982. 
[3] V. Abeles, S. Harrus, J. M. Angles et al., "Hypertrophic osteodystrophy in six weimaraner puppies associated with systemic signs," The Veterinary Record, vol. 145, no. 5, pp. 130-134, 1999.

[4] J. Grondalen, "Metaphyseal osteopathy (hypertrophic osteodystrophy) in growing dogs: a clinical study," Journal of Small Animal Practice, vol. 17, no. 11, pp. 721-735, 1976.

[5] S. Harrus, T. Waner, I. Aizenberg et al., "Development of hypertrophic osteodystrophy and antibody response in a litter of vaccinated Weimaraner puppies," Journal of Small Animal Practice, vol. 43, no. 1, pp. 27-31, 2002.

[6] E. LaFond, G. J. Breur, and C. C. Austin, "Breed susceptibility for developmental orthopedic diseases in dogs," Journal of the American Animal Hospital Association, vol. 38, no. 5, pp. 467477, 2002.

[7] A. P. Mee, M. T. Gordon, C. May, D. Bennett, D. C. Anderson, and P. T. Sharpe, "Canine distemper virus transcripts detected in the bone cells of dogs with metaphyseal osteopathy," Bone, vol. 14, no. 1, pp. 59-67, 1993.

[8] N. Safra, E. G. Johnson, L. Lit et al., "Clinical manifestations, response to treatment, and clinical outcome for Weimaraners with hypertrophic osteodystrophy: 53 cases (2009-2011)," Journal of the American Veterinary Medical Association, vol. 242, no. 9, pp. 1260-1266, 2013.

[9] K. S. Schulz, J. T. Payne, and E. Aronson, "Escherichia coli bacteremia associated with hypertrophic osteodystrophy in a dog," Journal of the American Veterinary Medical Association, vol. 199, no. 9, pp. 1170-1173, 1991.

[10] C. Adagra, D. Spielman, A. Adagra, and D. J. Foster, "Metaphyseal osteopathy in a British shorthair cat," Journal of Feline Medicine and Surgery, vol. 17, no. 4, pp. 367-370, 2015.

[11] F. Martínez, X. Manteca, and J. Pastor, "Retrospective study of morbidity and mortality of captive Iberian lynx (Lynx pardinus) in the ex situ conservation programme (2004-June 2010)," Journal of Zoo and Wildlife Medicine, vol. 44, no. 4, pp. 845-852, 2013.

[12] J. Queen, D. Bennett, S. Carmichael et al., "Femoral neck metaphyseal osteopathy in the cat," The Veterinary Record, vol. 142, no. 7, pp. 159-162, 1998.

[13] B. T. Kelly, A. Beyer, and M. K. Phillips, "Canis rufus," The IUCN Red List of Threatened Species Version 2014.2, 2014, http://www .iucnredlist.org.

[14] J. A. Teare, "Rhinoceros unicornis, conventional American units 2013," in ISIS Physiological Reference Intervals for Captive Wildlife: A CD-ROM Resource, International Species Information System, Bloomington, Minn, USA, 2013.

[15] D. R. Scanlan, M. A. Smith, H. D. Isenberg, S. Engrassia, and E. Hilton, "Clostridium bifermentans bacteremia with metastatic osteomyelitis," Journal of Clinical Microbiology, vol. 32, no. 11, pp. 2867-2868, 1994.

[16] T. A. Munjar, C. C. Austin, and G. J. Breur, "Comparison of risk factors for hypertrophic osteodystrophy," Veterinary and Comparative Orthopaedics and Traumatology, vol. 11, pp. 37-43, 1998.

[17] R. A. Read and G. M. Robins, "Deformity of the proximal tibia in dogs," Veterinary Record, vol. 111, no. 13, pp. 295-298, 1982.

[18] K. Dämmrich, "Relationship between nutrition and bone growth in large and giant dogs," Journal of Nutrition, vol. 121, pp. 114s-121s, 1991.

[19] A. Hedhammer, F. Wu, and L. Krook, "Overnutrition and skeletal disease," Cornell Veterinarian, vol. 64, supplement 1, pp. 128-135, 1974.
[20] W. Waddell, "Population analysis and breeding and transfer plan," Red Wolf (Canis rufus gregoryi) AZA Species Survival Plan Program, 2010.

[21] J. L. Paradiso and R. M. Nowak, "Canis rufus," Mammalian Species, no. 22, pp. 1-4, 1972.

[22] National Research Council ad hoc Committee on Dog and Cat Nutrition, Nutrient Requirements of Dogs and Cats, National Academies Press, Washington, DC, USA, 2006.

[23] W. Baumgärtner, R. W. Boyce, S. Alldinger et al., "Metaphyseal bone lesions in young dogs with systemic canine distemper virus infection," Veterinary Microbiology, vol. 44, no. 2-4, pp. 201-209, 1995.

[24] W. Baumgärtner, R. W. Boyce, S. E. Weisbrode, S. Aldinger, M. K. Axthelm, and S. Krakowka, "Histologic and immunocytochemical characterization of canine distemper-associated metaphyseal bone lesions in young dogs following experimental infection," Veterinary Pathology, vol. 32, no. 6, pp. 702-709, 1995. 

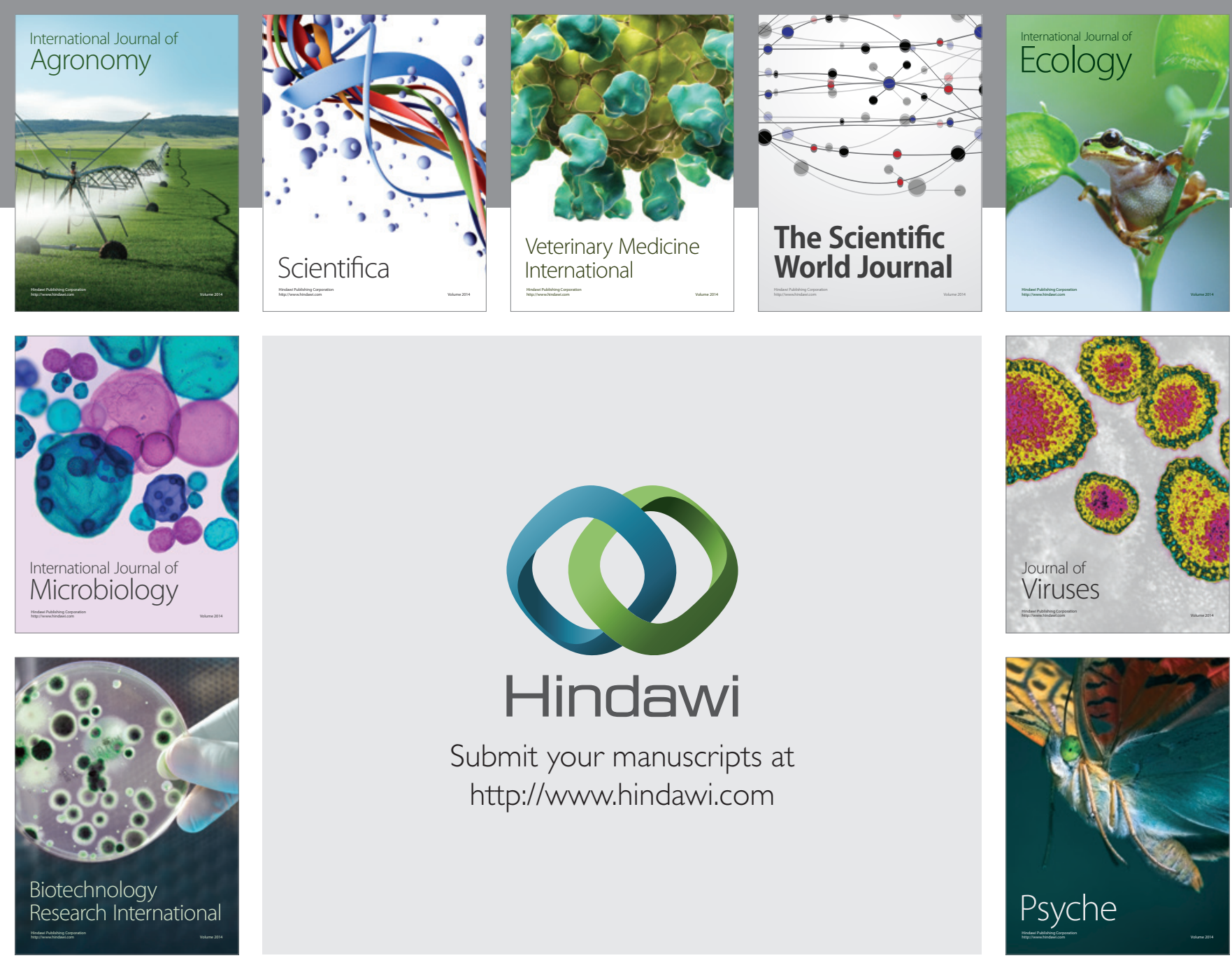

Submit your manuscripts at http://www.hindawi.com
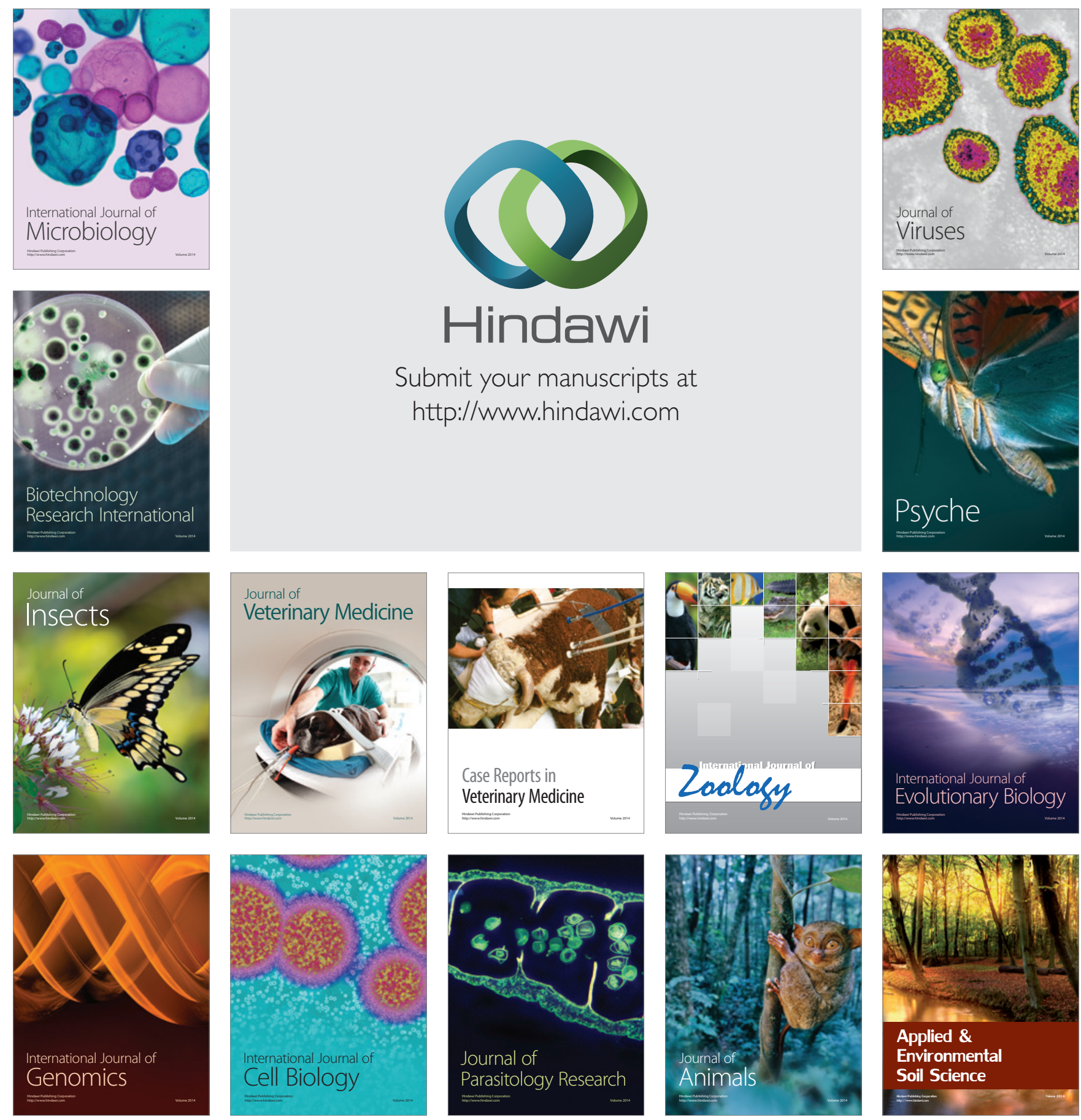